\title{
Effect of symmetry in the many-particle Wigner function
}

\author{
Emiliano Cancellieri, Paolo Bordone, and Carlo Jacoboni \\ Dipartimento di Fisica, Università di Modena e Reggio Emilia, and CNR-INFM S3 National Research Center, via Campi 213/a, \\ 41100 Modena, Italy
}

(Received 28 February 2007; revised manuscript received 30 August 2007; published 4 December 2007)

\begin{abstract}
An analysis of the Wigner function for identical particles is presented. Four situations have been considered. (i) The first is scattering process between two indistinguishable particles described by a minimum uncertainty wave packets showing the exchange and correlation effects in Wigner phase space. (ii) An equilibrium ensemble of $N$ particles in a one-dimensional box and in a one-dimensional harmonic potential is considered second, showing that the reduced one-particle Wigner function, as a function of the energy defined in the Wigner phase space, tends to the Fermi-Dirac or to the Bose-Einstein distribution function, depending on the considered statistics. (iii) The third situation is reduced one-particle transport equation for the Wigner function, in the case of interacting particles, showing the need for the two-particle reduced Wigner function within the Bogoliubov-Born-Green-Kirkwood-Yvon hierarchy scheme. (iv) Finally, the electron-phonon interaction in the two-particle case is considered, showing coparticipation of two electrons in the interaction with the phonon bath.
\end{abstract}

DOI: 10.1103/PhysRevB.76.214301

PACS number(s): 72.10.-d, 63.20.-e, 05.30.Fk

\section{INTRODUCTION}

Highly sophisticated technologies produce physical systems, and in particular semiconductor devices, of very small dimensions, comparable with the electron wavelength or with the electron coherence length. Under such conditions, semiclassical dynamics is not justified in principle, and interference effects due to the linear superpositions of quantum states have to be considered. Among the possible different approaches, the Wigner function (WF) has proved to be very useful for studying quantum electron transport, ${ }^{1-4}$ owing to its strong analogy with the semiclassical picture, since it explicitly refers to variables defined in an $(\mathbf{r}, \mathbf{p})$ Wigner phase space, together with a rigorous description of electron dynamics in quantum terms.

In this work we present an analysis of the WF for identical particles. Even though the WF was defined from its very beginning for the study of many-particle physics, in electron transport theory it has been used mainly in its one-particle version. The importance of the many-body problem derives from the fact that any real physical system one can think of is composed of a set of interacting bodies. Moreover, since we are dealing with quantum mechanical systems, the symmetry properties that describe the behavior of identical particles play an essential role. The present paper will be focused on the effects due to the symmetry of the spatial part of the wave function. The spin variables are not explicitly included. If particles with spin $1 / 2$ are considered (such as, e.g., electrons), a symmetric spatial wave function will correspond to an antisymmetric spin wave function and an antisymmetric spatial wave function will correspond to a symmetric spin wave function.

Four situations will be analyzed: (i) a scattering process between two indistinguishable particles described by minimum-uncertainty wave packets, showing the exchange and correlation effects in Wigner phase space; (ii) an equilibrium ensemble of $N$ particles in a box and in a harmonic potential, showing that the value of the WF in points in the
Wigner phase space with given energy tends to a FermiDirac (FD) or to a Bose-Einstein (BE) distribution function, depending on the considered statistics; (iii) the transport equation for interacting particles, showing the BogoliubovBorn-Green-Kirkwood-Yvon (BBGKY) hierarchy when the integral, over the degrees of freedom of all the particles but one, is performed, 5,6 (iv) the electron-phonon interaction in the case of two particles, where new Keldysh diagrams ${ }^{7}$ appear with respect to the one-electron case. ${ }^{8}$

\section{WIGNER FUNCTION FOR MANY IDENTICAL PARTICLES}

The WF was introduced by Wigner in 1932 to study quantum corrections to classical statistical mechanics. ${ }^{1,9-11}$ Thus, even though it is now used mainly in single-particle problems, from the very beginning this function was defined for $N$ particles as

$$
\begin{aligned}
f_{W}\left(\mathbf{r}_{1}, \mathbf{p}_{1}, \ldots, \mathbf{r}_{N}, \mathbf{p}_{N}, t\right)= & \int d \mathbf{s}_{1} \cdots d \mathbf{s}_{N} \exp \left(-\frac{i}{\hbar} \sum \mathbf{s}_{i} \mathbf{p}_{i}\right) \\
& \times \psi\left(\mathbf{r}_{1}+\frac{\mathbf{s}_{1}}{2}, \ldots, \mathbf{r}_{N}+\frac{\mathbf{s}_{N}}{2}, t\right) \\
& \times \psi^{\star}\left(\mathbf{r}_{1}-\frac{\mathbf{s}_{1}}{2}, \ldots, \mathbf{r}_{N}-\frac{\mathbf{s}_{N}}{2}, t\right) .
\end{aligned}
$$

In the case of identical particles, the wave function describing the many-body system satisfies well-known symmetry relations. When the position coordinates of two particles are interchanged, the spatial part of the wave function remains unaffected (in the symmetric case) or changes sign (in the antisymmetric case). Since the WF is bilinear in the wave function, it remains the same if the positions and, accordingly, the Wigner momenta of two particles are exchanged.

This symmetry property of the WF allows the definition of a reduced $M$-particle WF in a system of $N$ particles as ${ }^{12,13}$ 


$$
\begin{aligned}
f_{W}^{(N)} & \left(\mathbf{r}_{1}, \mathbf{p}_{1}, \ldots, \mathbf{r}_{M}, \mathbf{p}_{M}, t\right) \\
& =\frac{N !}{(N-M) ! h^{3(N-M)}} \int d \mathbf{r}_{M+1} d \mathbf{p}_{M+1} \cdots d \mathbf{r}_{N} d \mathbf{p}_{N} \\
& \times f_{W}\left(\mathbf{r}_{1}, \mathbf{p}_{1}, \ldots, \mathbf{r}_{N}, \mathbf{p}_{N}, t\right),
\end{aligned}
$$

where the superscript $(N)$ indicates that the reduced $M$-particle WF is defined in a system with $N$ particles. Note that in the case where $M=1$ the above equation becomes

$$
\begin{aligned}
f_{W}^{(N)}\left(\mathbf{r}_{1}, \mathbf{p}_{1}, t\right)= & \frac{N}{h^{3(N-1)}} \int d \mathbf{r}_{2} d \mathbf{p}_{2} \cdots d \mathbf{r}_{N} d \mathbf{p}_{N} \\
& \times f_{W}\left(\mathbf{r}_{1}, \mathbf{p}_{1}, \ldots, \mathbf{r}_{N}, \mathbf{p}_{N}, t\right) .
\end{aligned}
$$

The factorials appearing in front of the integral in Eq. (2) simplify to $N$ in Eq. (3), since this is the number of equivalent ways one can reduce the $N$-particle WF when the particles themselves are supposed to be identical.

\section{A. The WF for many single-particle wave functions}

We consider the case of $N$ particles in the system, and we define the WF with a wave function that is a symmetric or antisymmetric linear combination of products of singleparticle wave functions $\psi_{i}(\mathbf{r})(i=1, \ldots, N)$ as

$$
\begin{aligned}
& \psi\left(\mathbf{r}_{1}, \ldots, \mathbf{r}_{N}\right)=\psi_{1}\left(\mathbf{r}_{1}\right) \psi_{2}\left(\mathbf{r}_{2}\right) \psi_{3}\left(\mathbf{r}_{3}\right) \cdots \psi_{N}\left(\mathbf{r}_{N}\right) \\
& \quad \pm \psi_{1}\left(\mathbf{r}_{2}\right) \psi_{2}\left(\mathbf{r}_{1}\right) \psi_{3}\left(\mathbf{r}_{3}\right) \cdots \psi_{N}\left(\mathbf{r}_{N}\right) \\
& \quad+\psi_{1}\left(\mathbf{r}_{2}\right) \psi_{2}\left(\mathbf{r}_{3}\right) \psi_{3}\left(\mathbf{r}_{1}\right) \cdots \psi_{N}\left(\mathbf{r}_{N}\right) \\
& \quad \pm \psi_{1}\left(\mathbf{r}_{3}\right) \psi_{2}\left(\mathbf{r}_{2}\right) \psi_{3}\left(\mathbf{r}_{1}\right) \cdots \psi_{N}\left(\mathbf{r}_{N}\right)+\cdots
\end{aligned}
$$

where the upper sign is for the symmetric case and the lower for the antisymmetric case. In the WF expression it is possible to identify two different types of terms. The first one is characterized by the product of single-particle WFs. In each of these contributions, from the different wave functions, $N$ WFs are obtained that are evaluated in a particular permutation of the variable indices as, for example, $f_{W_{1}}\left(\mathbf{r}_{4}, \mathbf{p}_{4}\right) f_{W_{2}}\left(\mathbf{r}_{1}, \mathbf{p}_{1}\right) f_{W_{3}}\left(\mathbf{r}_{2}, \mathbf{p}_{2}\right) \ldots f_{W_{N}}\left(\mathbf{r}_{N-5}, \mathbf{p}_{N-5}\right)$.

The second type of contribution accounts for the exchange effects and vanishes when the wave functions $\psi_{n}(\mathbf{r})$ do not overlap. These terms are constituted by integrals of the product of $N$ factors $\psi_{n} \psi_{n}^{\star}$, one for each of the $N$ wave functions $\psi_{n}$. In these terms at least two products $\psi_{n}\left(\mathbf{r}_{i}\right.$ $\left.+\mathbf{s}_{i} / 2\right) \psi_{n}^{\star}\left(\mathbf{r}_{j}-\mathbf{s}_{j} / 2\right)$ are evaluated with $i \neq j$. It is the presence of such factors that makes it impossible to obtain the manyparticle WF in terms of single-particle WFs. The number of factors $\psi_{n} \psi_{n}^{\star}$, where $\psi_{n}$ and $\psi_{n}^{\star}$ correspond to different particles, appearing in a given integral can range from 2 to $N$. As an example, the WF in the case of $N=2$ reads

$$
\begin{aligned}
f_{W}\left(\mathbf{r}_{1}, \mathbf{p}_{1}, \mathbf{r}_{2}, \mathbf{p}_{2},\right)= & f_{W 1}\left(\mathbf{r}_{1}, \mathbf{p}_{1}\right) f_{W 2}\left(\mathbf{r}_{2}, \mathbf{p}_{2}\right)+f_{W 1}\left(\mathbf{r}_{2}, \mathbf{p}_{2}\right) f_{W 2}\left(\mathbf{r}_{1}, \mathbf{p}_{1}\right) \pm \frac{1}{\hbar^{6}} \int d \mathbf{s}_{1} d \mathbf{s}_{2} e^{(-i / \hbar)\left(\mathbf{s}_{1} \mathbf{p}_{1}+\mathbf{s}_{2} \mathbf{p}_{2}\right)} \\
& \times\left[\psi_{1}\left(\mathbf{r}_{1}+\frac{\mathbf{s}_{1}}{2}\right) \psi_{1}^{\star}\left(\mathbf{r}_{2}-\frac{\mathbf{s}_{2}}{2}\right) \psi_{2}\left(\mathbf{r}_{2}+\frac{\mathbf{s}_{2}}{2}\right) \psi_{2}^{\star}\left(\mathbf{r}_{1}-\frac{\mathbf{s}_{1}}{2}\right)+\psi_{1}\left(\mathbf{r}_{2}+\frac{\mathbf{s}_{2}}{2}\right) \psi_{1}^{\star}\left(\mathbf{r}_{1}-\frac{\mathbf{s}_{1}}{2}\right) \psi_{2}\left(\mathbf{r}_{1}+\frac{\mathbf{s}_{1}}{2}\right) \psi_{2}^{\star}\left(\mathbf{r}_{2}-\frac{\mathbf{s}_{2}}{2}\right)\right] ;
\end{aligned}
$$

here four terms appear, two for each kind of contribution. The two-particle system is treated in detail in Ref. 14.

\section{B. Example of two colliding particles}

A one-dimensional situation where two charged particles collide with each other has been simulated. The Schrödinger equation was solved with initial conditions given by two minimum-uncertainty wave packets interacting through the Coulomb potential, and the WF was evaluated at different time steps.

In Fig. 1 we plot the one-particle reduced WF of the system for the case of two Gaussian wave packets with opposite central wave vectors. Since we are dealing with a onedimensional system, the two particles are expected to decelerate, scatter, and then move away from each other. At $t=0$ we suppose the two particles to be described by an antisymmetric wave function. In Fig. 1(b) the system is shown 12 ps after the Coulomb interaction is switched on. At the beginning of the scattering process the exchange hole due to the
Pauli exclusion principle appears. In Fig. 1(c) the two particles are shown when their mutual distance has reached the minimum value. In this case the exchange hole is maximally evident. When the two particles are moving far enough from each other the exchange hole tends to disappear [Fig. 1(d)].

For the sake of comparison in Fig. 2 the evolution of the above system is represented in the case where the two particles at $t=0$ are described by a symmetric wave function. In this case, at $t=24 \mathrm{ps}$ we do not see the appearance of any exchange hole.

\section{EQUILIBRIUM WF FOR NONINTERACTING PARTICLES IN CONFINING POTENTIALS}

In this section a system of $N$ particles in two different confining potentials $V(\mathbf{r})$ has been studied. In the noninteracting case, the one-particle reduced WF is studied at thermal equilibrium at different temperatures. In order to simplify the mathematical treatment we shall introduce the 

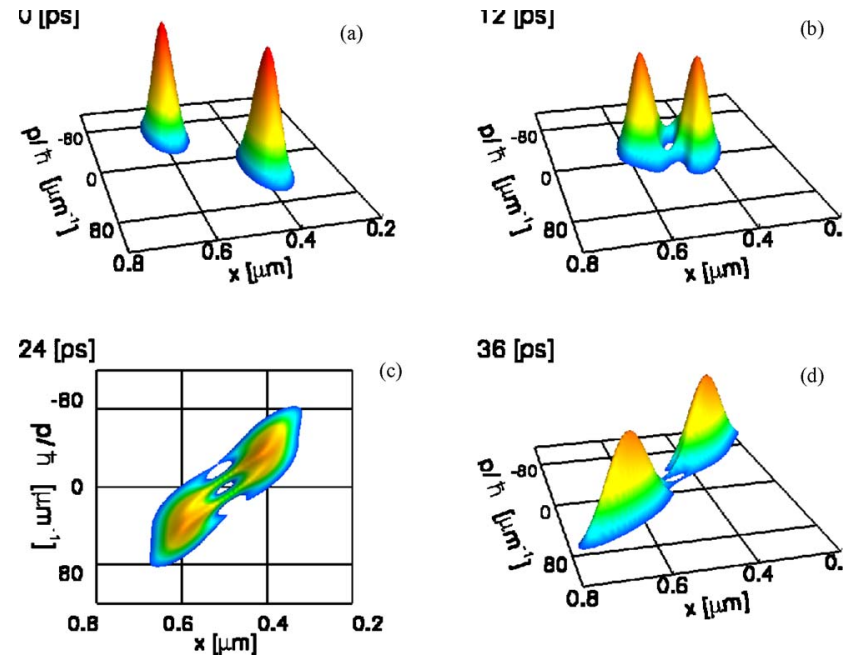

FIG. 1. (Color online) One-dimensional reduced one-particle WF of two interacting particles at different times. The case of an antisymmetric wave function, as initial condition, has been considered. The figure clearly shows the exchange hole due to the Pauli exclusion principle.

second-quantization notation. The $N$-particle wave function can thus be written as

$$
\psi\left(\mathbf{r}_{1}, \ldots, \mathbf{r}_{N}\right)=\left\langle\mathbf{r}_{1}, \ldots, \mathbf{r}_{N} \mid \psi\right\rangle=\left\langle 0\left|\hat{\Psi}\left(\mathbf{r}_{1}\right) \cdots \hat{\Psi}\left(\mathbf{r}_{N}\right)\right| \psi\right\rangle,
$$

and the WF as

$$
\begin{aligned}
f_{W}^{(N)}\left(\mathbf{r}_{1}, \mathbf{p}_{1}\right)= & \frac{N}{h^{3(N-1)}} \int d \mathbf{r}_{2} d \mathbf{p}_{2} \cdots d \mathbf{r}_{N} d \mathbf{p}_{N} \int d \mathbf{s}_{1} \cdots d \mathbf{s}_{N} \\
& \times \exp \left(-\frac{i}{\hbar} \sum_{j=1}^{N} \mathbf{p}_{j} \mathbf{s}_{j}\right)\langle 0| \hat{\Psi}\left(\mathbf{r}_{1}+\frac{\mathbf{s}_{1}}{2}\right) \\
& \cdots \hat{\Psi}\left(\mathbf{r}_{N}+\frac{\mathbf{s}_{N}}{2}\right)|\psi\rangle\langle\psi| \hat{\Psi}^{\dagger}\left(\mathbf{r}_{N}-\frac{\mathbf{s}_{N}}{2}\right) \\
& \cdots \hat{\Psi}^{\dagger}\left(\mathbf{r}_{1}-\frac{\mathbf{s}_{1}}{2}\right)|0\rangle
\end{aligned}
$$

where, here and in the following, $\hat{\Psi}$ and $\hat{\Psi}^{\dagger}$ are the creation

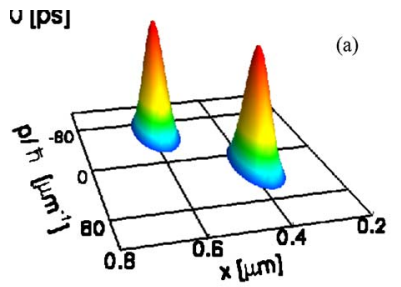

12 lps]
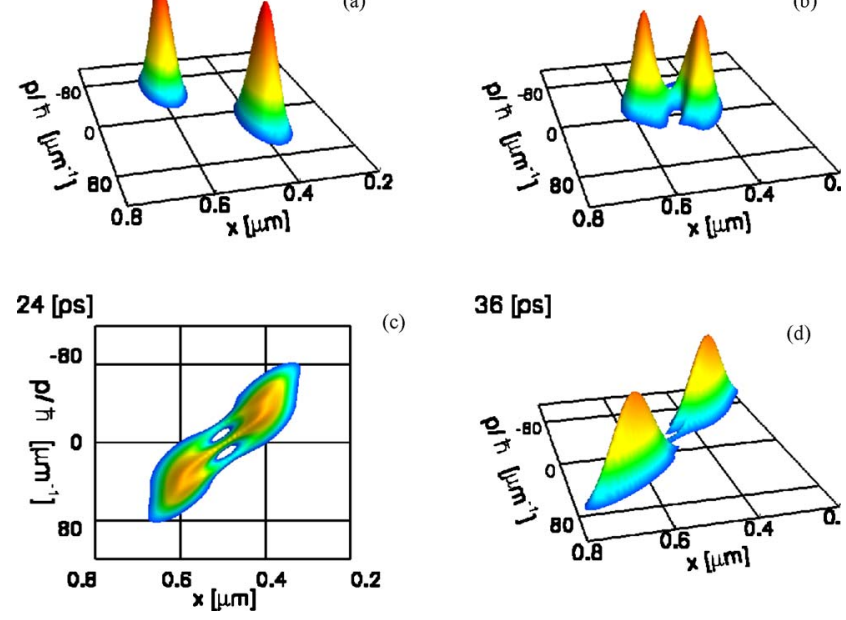

36 [ps]

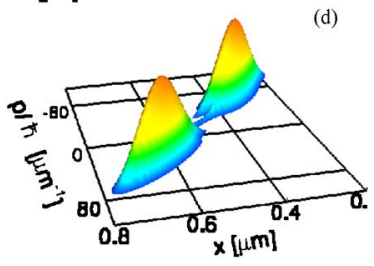

FIG. 2. (Color online) One-dimensional reduced one-particle WF of two interacting particles at different times. The case of a symmetric wave function, as initial condition, has been considered. The figure clearly shows that no exchange hole appears.

and annihilation field operators. Since we are interested in the thermal equilibrium distribution of a fixed number of particles, the density matrix in the above equation is

$$
\overline{|\psi\rangle\langle\psi|}=\hat{\rho}=\frac{1}{\mathcal{Z}} e^{-\hat{H} / k_{B} T}
$$

where $\mathcal{Z}$ is the partition function, $\hat{H}$ the Hamiltonian, $k_{B}$ the Boltzmann constant, and $T$ the temperature of the system. Since the particles in the system are supposed to be noninteracting, and the system is supposed to be confined, the Hamiltonian in its second-quantization form can easily be written in terms of the particle creation $\hat{c}_{n}^{\dagger}$ and annihilation $\hat{c}_{n}$ operators as

$$
\hat{H}=\sum_{n=1}^{\infty} \epsilon_{n} \hat{c}_{n}^{\dagger} \hat{c}_{n},
$$

where $\epsilon$ is the energy of the $n$th discrete level. Writing the field operators in terms of the creation and annihilation operators, the mean value element appearing in Eq. (7) becomes

$$
\begin{aligned}
\langle 0|\cdots| 0\rangle= & \sum_{n_{1}^{\prime}} \cdots \sum_{n_{N}^{\prime}} \sum_{n_{1}^{\prime \prime}} \cdots \sum_{n_{N}^{\prime \prime}}\left\langle 0\left|\hat{c}_{n_{1}^{\prime}} \cdots \hat{c}_{n_{N}^{\prime}} \exp \left(-\frac{1}{k_{B} T} \sum_{n} \epsilon_{n} \hat{c}_{n}^{\dagger} \hat{c}_{n}\right) \hat{c}_{n_{N}^{\prime \prime}}^{\dagger} \cdots \hat{c}_{n_{1}^{\prime \prime}}^{\dagger}\right| 0\right\rangle \psi_{n_{1}^{\prime}}\left(\mathbf{r}_{1}+\frac{\mathbf{s}_{1}}{2}\right) \cdots \psi_{n_{N}^{\prime}}\left(\mathbf{r}_{N}+\frac{\mathbf{s}_{N}}{2}\right) \\
& \times \psi_{n_{N}^{\prime \prime}}^{\star}\left(\mathbf{r}_{N}-\frac{\mathbf{s}_{N}}{2}\right) \cdots \psi_{n_{1}^{\prime \prime}}^{\star}\left(\mathbf{r}_{1}-\frac{\mathbf{s}_{1}}{2}\right)=\sum_{n_{1}^{\prime}} \cdots \sum_{n_{N}^{\prime}} \sum_{n_{1}^{\prime \prime}} \cdots \sum_{n_{N}^{\prime \prime}}\left\langle 0\left|\hat{c}_{n_{1}^{\prime}} \cdots \hat{c}_{n_{N}^{\prime}} \hat{c}_{n_{N}^{\prime \prime}}^{\dagger} \cdots \hat{c}_{n_{1}^{\prime \prime}}^{\dagger}\right| 0\right\rangle e^{-\left(1 / k_{B} T\right)\left(\epsilon_{n_{1}^{\prime \prime}}+\cdots+\epsilon_{n_{N}^{\prime \prime}}\right)} \\
& \times \psi_{n_{1}^{\prime}}\left(\mathbf{r}_{1}+\frac{\mathbf{s}_{1}}{2}\right) \cdots \psi_{n_{N}^{\prime}}\left(\mathbf{r}_{N}+\frac{\mathbf{s}_{N}}{2}\right) \psi_{n_{N}^{\prime \prime}}^{\star}\left(\mathbf{r}_{N}-\frac{\mathbf{s}_{N}}{2}\right) \cdots \psi_{n_{1}^{\prime \prime}}^{\star}\left(\mathbf{r}_{1}-\frac{\mathbf{s}_{1}}{2}\right),
\end{aligned}
$$




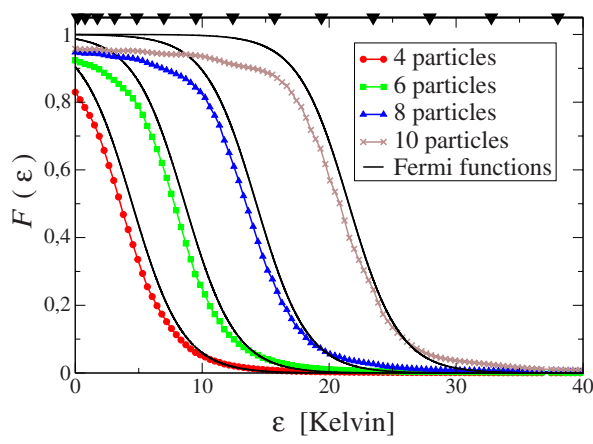

FIG. 3. (Color online) Average values $\mathcal{F}(\epsilon)$ of the points corresponding to the same energy interval. The case of a one-particle reduced WF of a system of $N$ particles with antisymmetric spatial part of the wave function, at thermal equilibrium $(T=2 \mathrm{~K})$, in onedimensional infinite square well potential is considered. Since the energy depends only upon the momenta of the particles, the average $\mathcal{F}(\epsilon)$ corresponds to the integral over the position variable $(x)$. The width of the well has been fixed to $150 \mathrm{~nm}$. In the upper part of the frame, the black triangles indicate the energies corresponding to the eigenstates of the well.

where $\psi_{n}$ indicates the $n$th eigenstate of the confining potential. As shown in the Appendix, Eq. (7) reduces to

$$
f_{W}^{(N)}\left(\mathbf{r}_{1}, \mathbf{p}_{1}\right)=\sum_{n_{1}^{\prime}} \bar{n}_{n_{1}^{\prime}} f_{W_{n_{1}^{\prime}}}\left(\mathbf{r}_{1}, \mathbf{p}_{1}\right) .
$$

In the limit of large $N$ and of an infinite number of allowed states with continuous energy spectrum, the above term gives the FD or the BE distribution function evaluated at an energy value $\epsilon_{n_{1}^{\prime}} \cdot{ }^{15}$

Once the one-particle reduced WF is obtained, the function $\mathcal{F}(\epsilon)$ giving the occupation number in an energy interval $\epsilon, \epsilon+\delta \epsilon$ can be evaluated as

$$
\mathcal{F}(\boldsymbol{\epsilon})=\frac{1}{V_{p s}(\boldsymbol{\epsilon})} \int_{\epsilon, \epsilon+\delta \epsilon} f_{W}^{(N)}\left(\mathbf{r}_{1}, \mathbf{p}_{1}\right) d \mathbf{r}_{1} d \mathbf{p}_{1},
$$

where $\boldsymbol{\epsilon}=\mathbf{p}_{1}^{2} / 2 m+V\left(\mathbf{r}_{1}\right)$ is the counterpart, in the Wigner phase space, of the classical energy. The integral is performed over those $\mathbf{r}_{1}$ and $\mathbf{p}_{1}$ giving a specific value of $\epsilon$ within the interval $\epsilon, \epsilon+\delta \epsilon$, and $V_{p s}(\epsilon)$ is the corresponding volume of the Wigner phase space.

\section{A. Examples for Fermi-Dirac statistics}

\section{Infinite square well potential}

An infinite square well potential in one dimension has been investigated at a temperature of $T=2 \mathrm{~K}$. In our simulations the width of the well has been kept constant to a value of $150 \mathrm{~nm}$. The one-particle reduced WF has been evaluated for $N=4,6,8$, and 10 by means of Eq. (11). Then $\mathcal{F}(\epsilon)$, the average values of the points of the WF corresponding to energy interval $\epsilon, \epsilon+\delta \epsilon$, have been plotted in Fig. 3 .

A comparison between our curves and the Fermi functions is obtained by evaluating the chemical potentials $\mu$ for $N$ $=4,6,8$, and 10 from a numerical solution of the equation

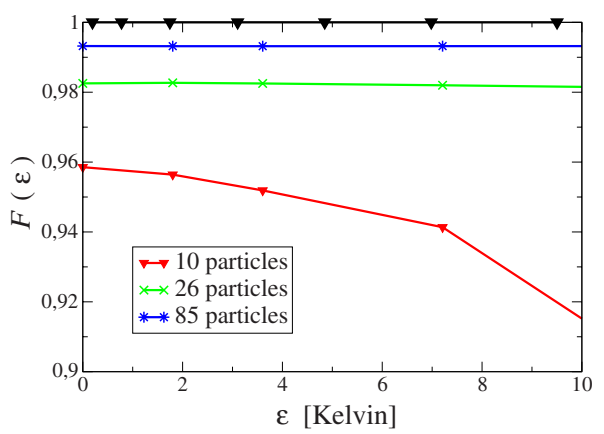

FIG. 4. (Color online) Average values $\mathcal{F}(\epsilon)$ of the points corresponding to the same energy of the one-particle reduced WF of a system of $N$ particles with antisymmetric spatial part of the wave function, at thermal equilibrium at a temperature of $2 \mathrm{~K}$, in a $1 \mathrm{D}$ infinite square well potential. The width of the well has been fixed to $150 \mathrm{~nm}$ and the number of fermions increased from 10 to 85 .

$$
N=\sum_{n=1}^{\infty} \frac{1}{e^{\left(\epsilon_{n}-\mu\right) / K_{B} T}+1} .
$$

The curves in Fig. 3 show a good agreement between the Fermi function and the average of the WF for any number of particles.

It is worth noting that, as expected, the agreement between the averages of WFs and the Fermi distributions is higher as the number of particles increases. However, in Fig. 3 , even in the case of ten particles the value of the WF's average corresponding to the point with $\epsilon=0$ does not reach the maximal value of 1 . For this reason we have plotted in Fig. 4 the system in more detail in the energy range from 0 to $10 \mathrm{~K}$ for a higher number of particles. As before, we simulate a well $150 \mathrm{~nm}$ wide with an electron gas at a temperature of $2 \mathrm{~K}$; in this case, however, the number of simulated particles is increased to 85 . Figure 4 shows that when the number of particles increases the value corresponding to $\epsilon$ $=0$ approaches 1 .

\section{Harmonic potential}

As a second example, we have studied a one-dimensional harmonic potential. Equation (11) has been evaluated for different numbers of particles (four, six, eight, and ten) at a temperature of $T=2 \mathrm{~K}$. The averages $\mathcal{F}(\epsilon)$ of the points of the WF belonging to the same energy interval $\epsilon, \epsilon+\delta \epsilon$ are plotted in Fig. 5. In the case of the harmonic potential this means

$$
\epsilon=\frac{p^{2}}{2 m}+\frac{1}{2} k x^{2},
$$

where $m$ is the mass of any particle in the system and $k$ is the spring constant. The Fermi function with the chemical potential given by Eq. (13) is clearly approached by the corresponding average of the WF.

\section{Effect of temperature versus level spacing}

It is possible to study how the particle distributions change when the dimension of the well or the strength of the 


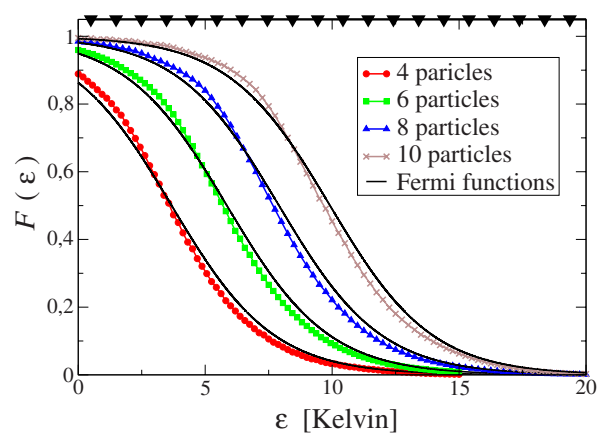

FIG. 5. (Color online) Average values $\mathcal{F}(\epsilon)$ of the points corresponding to the same energy of the reduced one-particle WF in a harmonic potential with spring constant $k=1.54 \times 10^{8} \mathrm{~kg} / \mathrm{s}^{2}$. The system in the case of $N=4,6,8,10$ particles is studied at thermal equilibrium at a temperature of $2 \mathrm{~K}$. The curves clearly tend to the FD distribution. In the upper part of the frame the black triangles indicate the energies corresponding to the eigenstates of the harmonic potential.

harmonic potential is varied. When the width of the well decreases or the strength of the harmonic potential increases, the spacing between the allowed energy levels increases and an oscillating behavior shows up in the curves (see Figs. 6 and 7). Our calculations have been performed in the case of a system with ten particles at a temperature of $2 \mathrm{~K}$. When the width of the well is decreased from 150 to $70 \mathrm{~nm}$, the energy gap between the ninth and the tenth energy levels increases from 3.8 to $17.1 \mathrm{~K}$. In the case of the harmonic potential, the strength of the force constant is varied from $1.54 \times 10^{8}$ to $5.69 \times 10^{8} \mathrm{~kg} / \mathrm{s}^{2}$, leading to an increase of the distance between the energy levels from 1 to $2 \mathrm{~K}$. The simulations show that such oscillations get more and more evident as the ratio between the spacing of the energy levels and $k_{B} T$ becomes greater. Under these conditions the approximation of a continuous spectrum of energies breaks down. Our outcomes indicate that, in the limit of large $N$ and of a continuous

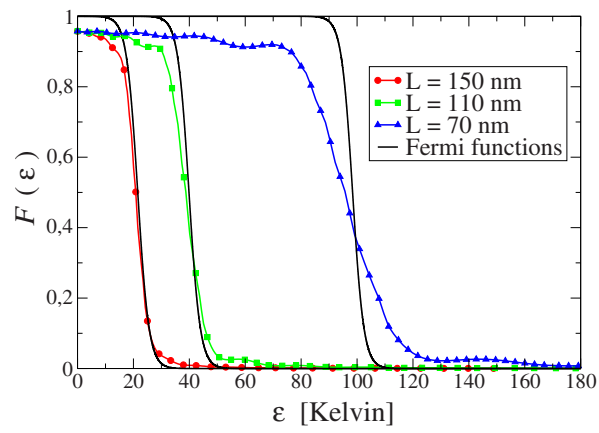

FIG. 6. (Color online) Average values $\mathcal{F}(\epsilon)$ of the points corresponding to the same energy of the reduced one-particle WF in a 1D infinite square potential well. In a system with ten particles with antisymmetric spatial part of the wave function at a temperature of $2 \mathrm{~K}$, the width of the well has been reduced from 150 to $70 \mathrm{~nm}$, which corresponds to an energy gap increase, from the ninth to the tenth energy levels, from 3.8 to $17.1 \mathrm{~K}$. When the energy spacing between the levels is bigger than the thermal energy, the particle distribution deviates from a Fermi function.

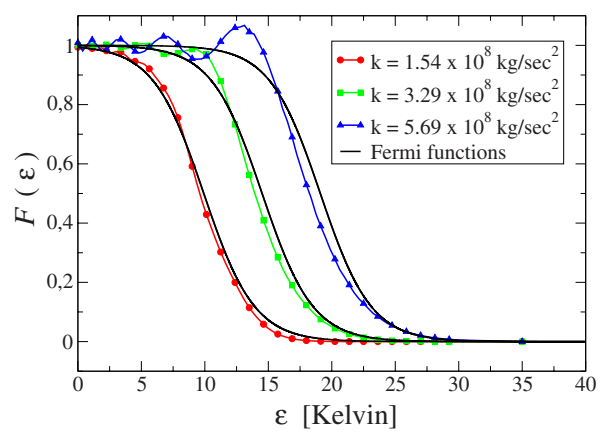

FIG. 7. (Color online) Average values $\mathcal{F}(\epsilon)$ of the points corresponding to the same energy interval of the reduced one-particle WF in a harmonic potential. In the case of ten particles with antisymmetric spatial part of the wave function the bound constant has been varied from $k=1.54 \times 10^{8}$ to $k=5.69 \times 10^{8} \mathrm{~kg} / \mathrm{s}^{2}$ corresponding to an increase of the spacing between the energy levels from 1 to $2 \mathrm{~K}$. When the gap between the energy levels increases and becomes bigger than $2 \mathrm{~K}$, the gas temperature, the particle distribution shows an oscillating behavior superimposed on the Fermi-like shape.

distribution of energy levels, the one-particle reduced WF reproduces the FD statistics.

\section{B. Examples for Bose-Einstein statistics}

\section{Infinite square potential well}

As in the previous Sec. III A, an infinite square well potential in one dimension has been investigated at a temperature of $T=2 \mathrm{~K}$. As before, the width of the well has been kept constant to a value of $150 \mathrm{~nm}$. The one-particle reduced WF has been evaluated in the case of $N=4$ and 10 particles by means of Eq. (11). Then $\mathcal{F}(\epsilon)$, the average values of the points of the WF corresponding to energy interval $\epsilon, \epsilon+\delta \epsilon$, have been plotted in Fig. 8. Finally, a comparison between our curves and the BE distribution functions is obtained, as in (13) by evaluating the chemical potentials $\mu$ for $N=4$ and 10 particles from a numerical solution of the following equation:

$$
N=\sum_{n=1}^{\infty} \frac{1}{e^{\left(\epsilon_{n}-\mu\right) / k_{B} T}-1} .
$$

\section{Harmonic potential}

As for the FD statistics we have studied, as a second example, a one-dimensional harmonic potential. Equation (11) has been evaluated for different numbers of particles (four and ten) at a temperature of $T=2 \mathrm{~K}$. The averages $\mathcal{F}(\epsilon)$ of the points of the WF belonging to the same energy interval $\epsilon, \epsilon+\delta \epsilon$ are plotted in Fig. 9. The Bose function with the chemical potential given by Eq. (15) is clearly approached by the corresponding average of the WF.

\section{Effect of temperature versus level spacing}

As a final example we study the effect of the temperature on the particle distribution and on the agreement between the 


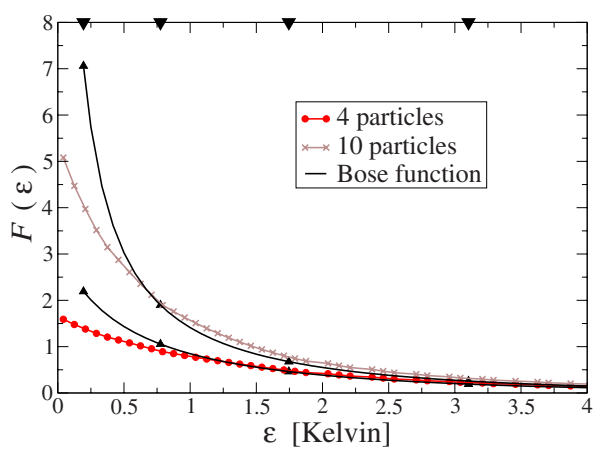

FIG. 8. (Color online) Average values $\mathcal{F}(\epsilon)$ of the points corresponding to the same energy interval. The case of a one-particle reduced WF of a system of $N$ particles with symmetric spatial part of the wave function, at thermal equilibrium $(T=2 \mathrm{~K})$, in a $1 \mathrm{D}$ infinite square well potential is considered. Since the energy depends only upon the momenta of the bosons, the average $\mathcal{F}(\epsilon)$ corresponds to the integral over the position variable $(x)$. The width of the well has been fixed to $150 \mathrm{~nm}$. In the upper part of the frame, the black triangles indicate the energies corresponding to the eigenstates of the well.

$\mathrm{BE}$ distribution functions and the curves obtained by means of our simulations. For both considered systems (i.e., the infinite square potential well and the harmonic potential) the agreement between the Bose distributions and our curves is improved by an increase of the equilibrium temperature. In Figs. 10 and 11 the well and the harmonic potential are studied in the case of ten particles. Our outcomes indicate that, for temperatures greater than or of the order of the level spacing, and in the limit of a continuous spectrum of energy levels, the one-particle reduced WF reproduces the BE distribution function.

\section{TRANSPORT EQUATION}

The dynamical equation for the single-particle WF is derived by differentiating the definition of the WF itself:

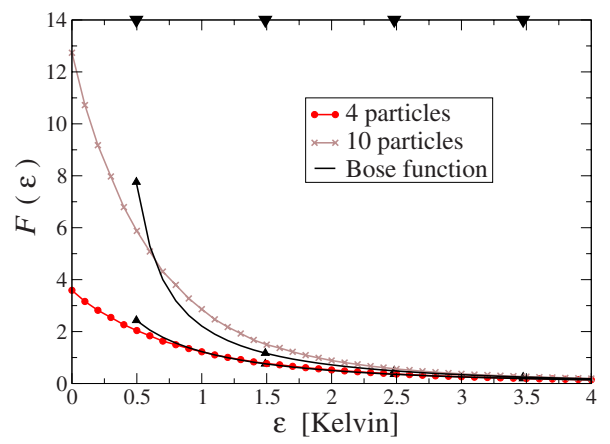

FIG. 9. (Color online) Average values $\mathcal{F}(\epsilon)$ of the points corresponding to the same energy of the reduced one-particle WF in a harmonic potential with spring constant $k=1.54 \times 10^{8} \mathrm{~kg} / \mathrm{s}^{2}$. The system in the case of $N=4$ and 10 particles with symmetric spatial part of the wave function is studied at thermal equilibrium at a temperature of $2 \mathrm{~K}$. The curves tend to the BE distribution. In the upper part of the frame, the black triangles indicate the energies corresponding to the eigenstates of the harmonic potential.

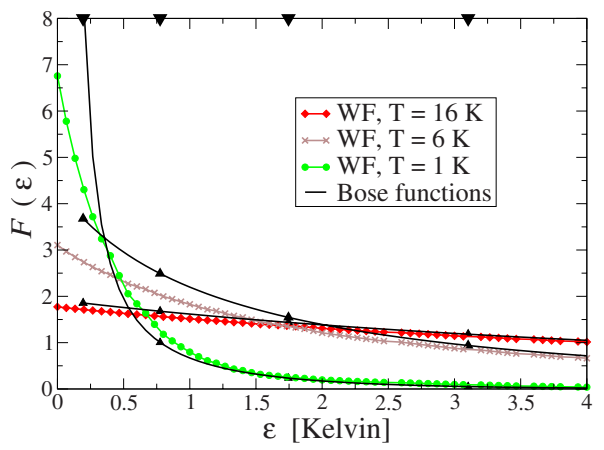

FIG. 10. (Color online) Average values $\mathcal{F}(\epsilon)$ of the points corresponding to the same energy of the reduced one-particle WF in a 1D infinite square potential well $150 \mathrm{~nm}$ wide. The system in the case of $N=10$ particles with symmetric spatial part of the wave function is studied at thermal equilibrium at a temperature varying from 1 to $16 \mathrm{~K}$. The agreement between our curves and the $\mathrm{BE}$ distribution functions clearly improves as the temperature increases. In the upper part of the frame, the black triangles indicate the energies corresponding to the eigenstates of the well.

$$
\frac{\partial}{\partial t} f_{W}(\mathbf{r}, \mathbf{p}, t)=\int d \mathbf{s} e^{-(i / \hbar)} \mathbf{s p} \frac{\partial}{\partial t}\left[\psi\left(\mathbf{r}+\frac{\mathbf{s}}{2}, t\right) \psi^{\star}\left(\mathbf{r}-\frac{\mathbf{s}}{2}, t\right)\right] .
$$

By means of the Schrödinger equation it is possible to evaluate the time derivative of the product of the two wave functions and to obtain the dynamical equation for the WF: ${ }^{4}$

$$
\begin{aligned}
\frac{\partial}{\partial t} f_{W}(\mathbf{r}, \mathbf{p}, t)= & -\frac{\mathbf{p}}{m} \nabla f_{W}(\mathbf{r}, \mathbf{p}, t)+\frac{1}{h^{3}} \int d \mathbf{p}^{\prime} \mathcal{V}_{W}\left(\mathbf{r}, \mathbf{p}-\mathbf{p}^{\prime}\right) \\
& \times f_{W}\left(\mathbf{r}, \mathbf{p}^{\prime}, t\right),
\end{aligned}
$$

where $\mathcal{V}_{W}$ is the interaction kernel for an external potential

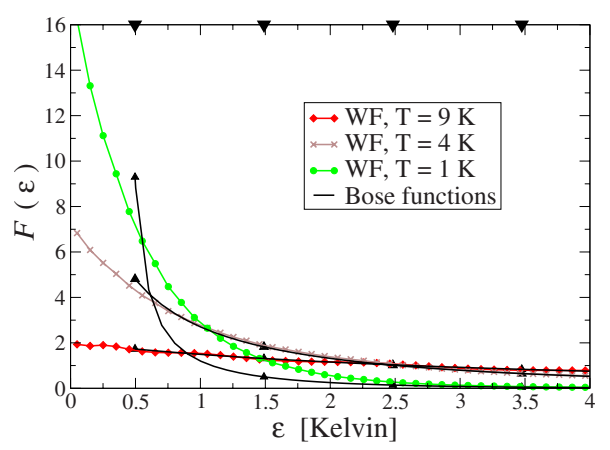

FIG. 11. (Color online) Average values $\mathcal{F}(\epsilon)$ of the points corresponding to the same energy of the reduced one-particle WF in a harmonic potential with spring constant $k=1.54 \times 10^{8} \mathrm{~kg} / \mathrm{s}^{2}$. The system in the case of $N=10$ particles with symmetric spatial part of the wave function is studied at thermal equilibrium at a temperature varying from 1 to $9 \mathrm{~K}$. The agreement between our curves and the $\mathrm{BE}$ distribution functions clearly improves as the temperature increases. In the upper part of the frame, the black triangles indicate the energies corresponding to the eigenstates of the harmonic potential. 
$V(\mathbf{r})$. Note that the interaction term, given by

$$
\mathcal{V}_{W}(\mathbf{r}, \mathbf{p})=\frac{1}{i \hbar} \int d \mathbf{s} e^{-(i / \hbar) \mathbf{p s}}\left[V\left(\mathbf{r}+\frac{\mathbf{s}}{2}\right)-V\left(\mathbf{r}-\frac{\mathbf{s}}{2}\right)\right]
$$

depends on the values of $V$ at points different from $\mathbf{r}$. However, while the nonlocality of $\mathcal{V}_{W}$ extends to infinity, its effect on the particle dynamics has to be considered only up to regions where the electron correlation is different from zero.

\section{A. Particle-particle scattering}

Let us study the transport equation for particle-particle scattering. In the case where no phonons nor external forces are present, the potential $V\left(\mathbf{r}_{1}, \mathbf{r}_{2}, \ldots, \mathbf{r}_{N}\right)$ is taken to be a Coulomb-like interaction and the transport equation thus reads

$$
\begin{aligned}
\frac{\partial}{\partial t} f_{W}\left(\mathbf{r}_{1}, \mathbf{p}_{1}, \ldots, \mathbf{r}_{N}, \mathbf{p}_{N}, t\right)= & -\sum_{l} \frac{\mathbf{p}_{l}}{m} \nabla_{\mathbf{r}_{l}} f_{W}\left(\mathbf{r}_{1}, \mathbf{p}_{1}, \ldots, \mathbf{r}_{l}, \mathbf{p}_{l}, \ldots, \mathbf{r}_{N}, \mathbf{p}_{N}, t\right)+\frac{1}{\hbar^{3}} \sum_{i} \sum_{j} \int d \mathbf{p}_{i}^{\prime} d \mathbf{p}_{j}^{\prime} \delta\left(\Delta \mathbf{p}_{i}+\Delta \mathbf{p}_{j}\right) V_{W}\left(\left|\mathbf{r}_{i}-\mathbf{r}_{j}\right|, \Delta \mathbf{p}_{i}\right. \\
& \left.-\Delta \mathbf{p}_{j}\right) \times f_{W}\left(\mathbf{r}_{1}, \mathbf{p}_{1}, \ldots, \mathbf{r}_{i}, \mathbf{p}_{i}^{\prime}, \ldots, \mathbf{r}_{j}, \mathbf{p}_{j}^{\prime}, \ldots \mathbf{r}_{N}, \mathbf{p}_{N}, t\right),
\end{aligned}
$$

where $V_{W}$ is the potential kernel of the Wigner equation and $\Delta \mathbf{p}=\mathbf{p}-\mathbf{p}^{\prime}$. As done before, in order to get a better understanding of the above equation, the kernel that describes the particle-particle interaction is studied for $N=2$ :

$$
V_{W}\left(\mathbf{r}_{1}, \mathbf{r}_{2}, \mathbf{p}_{1}, \mathbf{p}_{2}\right)=\frac{1}{i \hbar} \int d \mathbf{s}_{1} d \mathbf{s}_{2} e^{-(i / \hbar)\left(\mathbf{p}_{1} \mathbf{s}_{1}+\mathbf{p}_{2} \mathbf{s}_{2}\right)} \times\left[V\left(\mathbf{r}_{1}+\frac{\mathbf{s}_{1}}{2}, \mathbf{r}_{2}+\frac{\mathbf{s}_{2}}{2}\right)-V\left(\mathbf{r}_{1}-\frac{\mathbf{s}_{1}}{2}, \mathbf{r}_{2}-\frac{\mathbf{s}_{2}}{2}\right)\right] .
$$

Since the Coulomb interaction depends only upon the distance between the two particles, it is useful to rewrite the above equation using the new variables $\mathbf{x}=\mathbf{r}_{1}-\mathbf{r}_{2}, \mathbf{s}=\mathbf{s}_{1}-\mathbf{s}_{2}$, and $\mathbf{s}^{\prime}=\left(\mathbf{p}_{1} \mathbf{s}_{2}+\mathbf{p}_{2} \mathbf{s}_{1}\right) /\left(\mathbf{p}_{1}+\mathbf{p}_{2}\right)$ :

$$
\begin{aligned}
V_{W}\left(\mathbf{r}_{1}, \mathbf{r}_{2}, \mathbf{p}_{1}, \mathbf{p}_{2}\right) & =\frac{1}{i \hbar} \int d \mathbf{s} d \mathbf{s}^{\prime} e^{-(i / \hbar)\left(\mathbf{p}_{1}+\mathbf{p}_{2}\right) \mathbf{s}^{\prime}} e^{-(i / \hbar)\left(\mathbf{p}_{1}-\mathbf{p}_{2}\right) \mathbf{s}}\left[V\left(\mathbf{x}+\frac{\mathbf{s}}{2}\right)-V\left(\mathbf{x}-\frac{\mathbf{s}}{2}\right)\right] \\
& =\frac{\hbar^{3}}{i \hbar} \delta\left(\mathbf{p}_{1}+\mathbf{p}_{2}\right) \int d \mathbf{s} e^{-(i / \hbar)\left(\mathbf{p}_{1}-\mathbf{p}_{2}\right) \mathbf{s}}\left[V\left(\mathbf{x}+\frac{\mathbf{s}}{2}\right)-V\left(\mathbf{x}-\frac{\mathbf{s}}{2}\right)\right]=\hbar^{3} \delta\left(\mathbf{p}_{1}+\mathbf{p}_{2}\right) V_{W}\left(\mathbf{r}_{1}-\mathbf{r}_{2}, \mathbf{p}_{1}-\mathbf{p}_{2}\right) .
\end{aligned}
$$

Thus the factor $\delta\left(\Delta \mathbf{p}_{i}+\Delta \mathbf{p}_{j}\right)$ appearing in Eq. (19) represents the constraint for the total momentum conservation, while the difference $\left(\Delta \mathbf{p}_{i}-\Delta \mathbf{p}_{j}\right)$ indicates that the interaction depends only upon the momentum transfer between particles $i$ and $j$.

When the one-particle reduced WF in the case of $N$ particles is evaluated, Eq. (19) reads

$$
\begin{aligned}
\frac{\partial}{\partial t} f_{W}^{(N)}(\mathbf{r}, \mathbf{p}, t)= & -\frac{\mathbf{p}}{m} \nabla_{\mathbf{r}} f_{W}^{(N)}(\mathbf{r}, \mathbf{p}, t)+\frac{1}{\hbar^{3}} \int d \varrho d \mathbf{p}_{\varrho} \int d \mathbf{p}^{\prime} \\
& \times V_{W}(|\mathbf{r}-\varrho|, 2 \Delta \mathbf{p}) f_{W}^{(N)}\left(\mathbf{r}, \mathbf{p}^{\prime}, \varrho, \mathbf{p}_{\varrho}, t\right),
\end{aligned}
$$

where $\mathbf{r}$ and $\mathbf{p}$ are the position and the Wigner momentum of the considered particle and, $\varrho$ and $\mathbf{p}_{\varrho}$ indicate the position coordinates of one of the remaining $N-1$ particles. It should be noticed that all the particles are interacting with each other, but, due to their indistinguishability, all the contributions are identical and sum up to balance the factorials appearing in Eq. (2). The above expression shows that the transport equation for the reduced one-particle WF depends on the reduced two-particle WF. When the transport equation for the reduced two-particle WF is evaluated, the particleparticle interaction term depends upon the three-particle reduced WF, and so on for the transport equation for the other reduced WFs. It is the Wigner picture of the BBGKY hierarchy.

When the WF is written in terms of antisymmetric singleparticle wave functions, as we have seen in Eq. (5), two types of contributions can be identified. It is then possible to study how the transport equation reads when only the contributions due to nonoverlapping wave functions are considered:

$$
\begin{aligned}
\frac{\partial}{\partial t} f_{W}^{(N)}(\mathbf{r}, \mathbf{p}, t)= & \frac{\partial}{\partial t} \sum_{i} f_{W_{i}}^{(N)}(\mathbf{r}, \mathbf{p}, t)=-\frac{\mathbf{p}}{m} \nabla_{\mathbf{r}} f_{W}^{(N)}(\mathbf{r}, \mathbf{p}, t) \\
& +\frac{1}{\hbar^{3}} \sum_{i} \int d \mathbf{p}^{\prime} d \varrho V_{W}(|\mathbf{r}-\varrho|, 2 \Delta \mathbf{p}) \\
& \times\left(\sum_{j \neq i}\left|\psi_{j}(\varrho)\right|^{2}\right) f_{W_{i}}^{(N)}\left(\mathbf{r}, \mathbf{p}^{\prime}, t\right) .
\end{aligned}
$$

In addition to a Liouvillian contribution, an interaction term appears where each one-particle contribution interacts with all the others as in the Hartree approximation. In the case of overlapping wave functions also the other kind of contributions [as studied in Eq. (5)] must be considered, and the exchange term is restored. 


\section{B. Electron-phonon scattering for the two-electron WF}

If two electrons are interacting with the phonon gas, the state of the system is described by the electron state and the state of the crystal vibrations. A proper basis set in this case can be

$$
\left|\mathbf{r}_{1}, \mathbf{r}_{2},\left\{n_{\mathbf{q}}\right\}\right\rangle,
$$

where $\left\{n_{\mathbf{q}}\right\}$ is the set of occupation numbers of the phonon modes q. In this case the electron-phonon (e-ph) interaction Hamiltonian is

$$
\mathcal{H}_{e p}=\sum_{\mathbf{q}} i \hbar F(\mathbf{q})\left[a_{\mathbf{q}}\left(e^{i \mathbf{q} \mathbf{r}_{1}}+e^{i \mathbf{q} \mathbf{r}_{2}}\right)-a_{\mathbf{q}}^{\dagger}\left(e^{-i \mathbf{q} \mathbf{r}_{1}}+e^{-i \mathbf{q} \mathbf{r}_{2}}\right)\right],
$$

where $a_{\mathbf{q}}$ and $a_{\mathbf{q}}^{\dagger}$ are the annihilation and creation operators for the phonon mode $\mathbf{q}$, with frequency $\omega_{\mathbf{q}}$ and $F(\mathbf{q})$ is a real function that depends on the $e$-ph interaction mechanism. In this case the $e$-ph interaction term for the dynamical equation for two electrons is made out of eight terms as follows:

$$
\begin{aligned}
\left.\frac{\partial}{\partial t} f_{W}\right|_{e p}= & \sum_{\mathbf{q}^{\prime}} F\left(\mathbf{q}^{\prime}\right)\left[e^{i\left[\mathbf{q}^{\prime} \mathbf{r}_{1}-\omega_{q^{\prime}}\left(t-t_{0}\right)\right]} \sqrt{n_{q^{\prime}}+1} f_{W}\left(\mathbf{r}_{1}, \mathbf{p}_{1}-\frac{\hbar \mathbf{q}^{\prime}}{2}, \mathbf{r}_{2}, \mathbf{p}_{2},\left\{\ldots, n_{q^{\prime}}+1, \ldots\right\},\left\{n_{q}^{\prime}\right\}, t\right)-e^{-i\left[\mathbf{q}^{\prime} \mathbf{r}_{1}-\omega_{q^{\prime}}\left(t-t_{0}\right)\right]} \sqrt{n_{q^{\prime}}}\right. \\
& \times f_{W}\left(\mathbf{r}_{1}, \mathbf{p}_{1}+\frac{\hbar \mathbf{q}^{\prime}}{2}, \mathbf{r}_{2}, \mathbf{p}_{2},\left\{\ldots, n_{q^{\prime}}-1, \ldots\right\},\left\{n_{q}^{\prime}\right\}, t\right)+e^{-i\left[\mathbf{q}^{\prime} \mathbf{r}_{1}-\omega_{q^{\prime}\left(t-t_{0}\right)}\right]} \sqrt{n_{q^{\prime}}^{\prime}+1} \\
& \times f_{W}\left(\mathbf{r}_{1}, \mathbf{p}_{1}-\frac{\hbar \mathbf{q}^{\prime}}{2}, \mathbf{r}_{2}, \mathbf{p}_{2},\left\{n_{q}\right\},\left\{\ldots, n_{q^{\prime}}^{\prime}+1, \ldots\right\}, t\right)-e^{i\left[\mathbf{q}^{\prime} \mathbf{r}_{1}-\omega_{q^{\prime}}\left(t-t_{0}\right)\right]} \sqrt{n_{q^{\prime}}^{\prime}} \\
& \left.\times f_{W}\left(\mathbf{r}_{1}, \mathbf{p}_{1}+\frac{\hbar \mathbf{q}^{\prime}}{2}, \mathbf{r}_{2}, \mathbf{p}_{2},\left\{n_{q}\right\},\left\{\ldots, n_{q^{\prime}}^{\prime}-1, \ldots\right\}, t\right)+\text { o.p. }\right]
\end{aligned}
$$

where $\mathbf{r}_{1}, \mathbf{p}_{1}, \mathbf{r}_{2}, \mathbf{p}_{2}$ are the Wigner phase-space coordinates of the two particles. In the above equation, o.p. stands for other particle and indicates the four terms where $\mathbf{r}_{2}$ replaces $\mathbf{r}_{1}$ in the exponential factors and $\mathbf{p}_{2}$ undergoes a variation of $\hbar \mathbf{q}^{\prime} / 2$ while $\mathbf{p}_{1}$ remains unchanged.

The eight terms appearing on the right-hand side (RHS) of the above equation have simple physical interpretations: the $e$-ph interaction occurs as emission or absorption of a quantum of any mode $\mathbf{q}$ and this may appear in the state on the left or on the right of the bilinear expression that defines the WF. Each elementary interaction or vertex changes only one of the two sets of variables of the WF; more precisely, one of the occupation numbers $n_{q}$ is changed by unity and one of the electron momenta is changed by half of the phonon momentum.

In analogy with the Chambers formulation ${ }^{16}$ of the classical kinetic equation, it is possible to introduce new variables $\left(\mathbf{r}_{i}^{*}, \mathbf{p}_{i}^{*}, t^{*}\right)$ that allow us to obtain an integral form of the dynamical equation for the WF. This integral equation is in a closed form and can be solved by iteratively substituting it into itself, leading to what is known as its Neumann expansion.

Equation (26) gives eight terms for the contribution of the first order of the Neumann expansion, 64 terms for the contribution of the second order, and so on for the higher-order terms. In order to obtain meaningful physical quantities, however, the trace over the phonon modes must be performed, leading to a vanishing contribution for each term corresponding to an odd order in the Neumann expansion. ${ }^{8}$ Only terms with an even number of vertices give diagonal (in the phonon modes) contributions different from zero.
As stated before, the second order in the Neumann expansion gives 64 terms. Among these, 32 yield contributions diagonal in the phonon modes and survive to the trace operation, 16 terms refer to one particle and 16 to the other. For each particle eight terms are the complex conjugate of the other eight and can be summed together, leading to eight contributions for each particle. Among these it is possible to recognize four standard interactions undergone by each particle: real emission, real absorption, virtual emission, and virtual absorption.

The main difference from the single-particle case lies in the eight (four for each particle) remaining terms. In the two-particle case the phonon occupation number can be changed, in the first or second set of values in the arguments of the WF, not just by one electron losing (gaining) a Wigner momentum equal to half the phonon momentum in each of the two vertices ${ }^{8}$ but also by the action of two electrons. One electron loses or gains half the phonon momentum in the first vertex and another electron loses or gains half the phonon momentum in the second vertex. Since we are dealing with identical particles, we do not know which electron interacts with the phonon bath in the first or in the second vertex. These four terms are real or virtual emissions or absorptions where the interaction with the phonon bath is shared between the two electrons.

It should be recalled that the $\mathbf{p}$ variable of the WF is obtained as a linear combination of two electron momenta. A specific value $\widetilde{\mathbf{p}}$ is obtained as $\widetilde{\mathbf{p}}=\hbar\left(\mathbf{k}_{1}+\mathbf{k}_{2}\right) / 2$ where $\mathbf{k}_{1}$ and $\mathbf{k}_{2}$ range from $-\infty$ to $+\infty$. For this reason the Wigner momentum undergoes a change corresponding to half of the phonon momentum at each interaction vertex. 


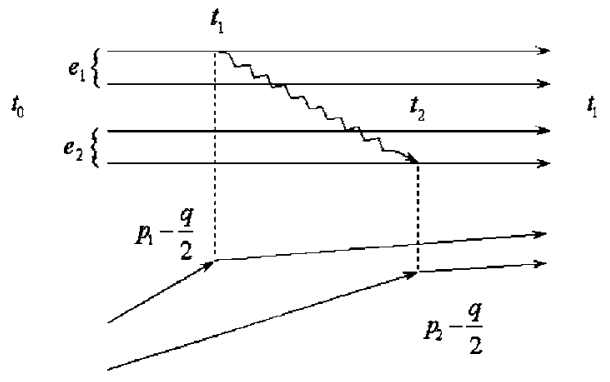

space the classical energy $\mathbf{p}^{2} / 2 m+V(\mathbf{r})$. The agreement improves as the spacing between the energy levels decreases.

\section{ACKNOWLEDGMENTS}

This work has been partially supported by the U.S. Office of Naval Research (Contract No. N00014-03-1-0289).

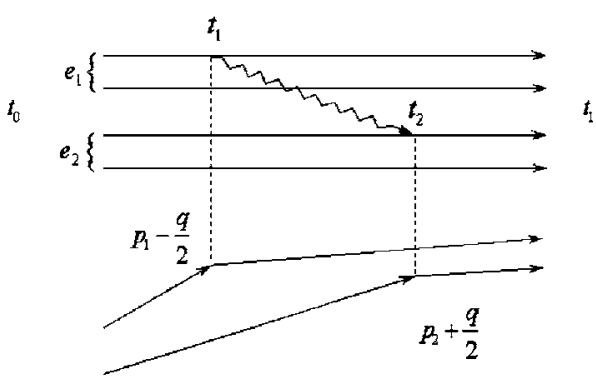

FIG. 12. Real emission of a phonon mode in mutual participation by two electrons. One electron $e_{1}$ changes its Wigner momentum by half of the phonon momentum at time $t=t_{1}$ and the other electron $e_{2}$ does the same at a later time $t=t_{2}$ (upper box). Virtual emission occurs where one electron lose a Wigner momentum equal to half the momentum of the phonon while the other electron gains the same amount (lower box).

In Fig. 12, by means of the Keldysh-diagram formalism, two of the coparticipating graphs are shown: a real phonon emission due to electron-electron cooperation and a virtual emission, respectively. In each case both the Keldysh diagram representing the transition and the corresponding Wigner path ${ }^{8}$ undergone by the two electrons are shown. Since the Keldysh diagrams are in the density matrix (DM) representation, four timelines appear, two for each electron. The first and the third lines correspond to the first wave function of the DM while the other two correspond to the second wave function. In the upper box at time $t=t_{1}$ one electron in the first wave function of the DM emits a phonon and at time $t=t_{2}$ another electron in the second wave function emits the same phonon.

In the lower box of Fig. 12 at time $t=t_{2}$ an electron absorbs the phonon emitted at $t=t_{1}$, corresponding to a virtual emission.

\section{CONCLUSION}

We have developed a model based on the WF formalism that allows us to introduce the symmetry effect in a system where particles interact with each other and with the phonon bath. We have shown how this formalism can be useful by applying it to different situations: the study of particleparticle scattering, of the thermal distribution of $N$ particles in confining potentials, and of the two-electron dynamics in the presence of electron-phonon scattering. One of the main results of this work is that the FD and $\mathrm{BE}$ distribution functions are reasonably reproduced using in the Wigner phase

\section{APPENDIX}

The study of the thermal equilibrium of a system of $N$ particles in a confining potential, by means of the manyparticle WF, has shown [see Eqs. (7) and (10)] that the oneparticle reduced WF can be written as a linear combination of the product of the single-particle wave functions of the eigenstates of the potential.

By means of the normalization condition, we prove that, when the WF is written in terms of Eqs. (7) and (10), it is possible to isolate $N-1$ summations that correspond to the average occupation number $\left(\bar{n}_{s}\right)$ of the $s$ th energy eigenstate, as defined in the study of quantum statistics of an ideal gas in Ref. 15. In the limit of a continuous spectrum of energy levels, the average number of particles $\bar{n}_{s}$ can be shown to reproduce the $\mathrm{FD}$ or the $\mathrm{BE}$ distribution function, according to the specific symmetry of the wave function. Moreover, we show that the many-particle WF can be written as a linear combination of the single-particle WFs of the eigenstates of the potential, and that the weights of such linear combination coincide with the average occupation numbers $\bar{n}_{s}$.

Following Ref. 15 the average number of particles in a given energy level is defined as

$$
\bar{n}_{S}=\frac{1}{\mathcal{Z}} \sum_{R} n_{s} e^{-\left(1 / k_{B} T\right) \epsilon_{R}},
$$

where $\mathcal{Z}$ is the partition function and is defined as

$$
\mathcal{Z}=\sum_{R} e^{-\left(1 / k_{B} T\right) \epsilon_{R}}
$$

The summation is extended over all the allowed configurations $R$ having energy $\epsilon_{R}$. In the first equation $n_{s}$ is the number of particles in the $s$ th level for a particular configuration. For a given configuration the argument of the summation reads

$$
n_{s} e^{-\left(1 / k_{B} T\right)\left(n_{1} \epsilon_{1}+n_{2} \epsilon_{2}+\cdots+n_{N} \epsilon_{N}\right)}
$$

where $\epsilon_{i}$ is the energy of the $i$ th eigenstate occupied by $n_{i}$ particles. Summing the average number of particles occupying the $s$ th state $\left(\bar{n}_{s}\right)$ over all the eigenstates, the total number $(N)$ of particles is obtained. Thus

$$
\sum_{s} \sum_{R} n_{s} e^{-\left(1 / k_{B} T\right) \epsilon_{R}}=N \mathcal{Z}
$$

Let us now consider the $N$-particle WF; for the sake of clarity we study the one-dimensional case. We start from the normalization condition for the WF: 


$$
\frac{1}{h^{N}} \int d x_{1} d p_{1} \cdots d x_{N} d p_{N} f_{W}\left(x_{1}, p_{1}, \ldots, x_{N}, p_{N}\right)=N
$$

then we consider the $N$-particle WF written in second quantization as given by Eqs. (7) and (10). In Eq. (A5), performing the integral over the variable $p_{i}$, a Dirac delta function $\delta\left(s_{i}\right)$ is obtained for every particle $i$ :

$$
\frac{1}{h} \int d p_{i} e^{-(i / \hbar) p_{i} s_{i}}=\delta\left(s_{i}\right)
$$

Then, performing the integral over all the variables $s_{i}$, each one-particle wave function $\psi_{n_{i}^{\prime}}\left(x_{i}+s_{1} / 2\right)$ and $\psi_{n_{i}^{\prime \prime}}^{\star}\left(x_{i}-s_{1} / 2\right)$ is evaluated at the point $x_{i}$ :

$$
\int d s_{i} \delta\left(s_{i}\right) \psi_{n_{i}^{\prime}}\left(x_{i}+\frac{s_{i}}{2}\right) \psi_{n_{i}^{\prime \prime}}^{\star}\left(x_{i}-\frac{s_{i}}{2}\right)=\psi_{n_{i}^{\prime}}\left(x_{i}\right) \psi_{n_{i}^{\prime \prime}}^{\star}\left(x_{i}\right) .
$$

Finally, for each particle, the integral over the variable $x_{i}$ gives a Kronecker delta function $\delta_{n_{i}^{\prime} n_{i}^{\prime \prime}}$ since the one-particle wave functions $\psi_{n_{i}^{\prime}}$ and $\psi_{n_{i}^{\prime \prime}}^{\star}$ are eigenstates of the confining potential:

$$
\int d x_{i} \psi_{n_{i}^{\prime}}\left(x_{i}\right) \psi_{n^{\prime \prime} s_{i}}^{\star}\left(x_{i}\right)=\delta_{n_{i}^{\prime} n_{i}^{\prime \prime}}
$$

The normalization condition can now be written as

$$
\begin{aligned}
& \frac{1}{h^{N}} \int d x_{1} d p_{1} \cdots d x_{N} d p_{N} f_{W}\left(x_{1}, p_{1}, \ldots, x_{N}, p_{N}\right) \\
& =\frac{1}{\mathcal{Z}} \sum_{n_{1}^{\prime}} \cdots \sum_{n_{N}^{\prime}} \sum_{n_{1}^{\prime \prime}} \cdots \sum_{n_{N}^{\prime \prime}}\left\langle 0\left|\hat{c}_{n_{1}^{\prime}} \cdots \hat{c}_{n_{N}^{\prime}} \hat{c}_{n_{N}^{\prime \prime}}^{\dagger} \ldots \hat{c}_{n_{1}^{\prime \prime}}^{\dagger}\right| 0\right\rangle \\
& \times e^{-\left(1 / k_{B} T\right)\left(\epsilon_{\left.n_{1}^{\prime \prime}+\cdots+\epsilon_{n_{N}^{\prime \prime}}\right)}\right.} \delta_{n_{1}^{\prime} n_{1}^{\prime \prime}} \cdots \delta_{n_{N}^{\prime} n_{N}^{\prime \prime}}
\end{aligned}
$$

Using Eq. (A5) and the Kronecker $\delta$ s to perform the summations over the indices $n_{i}^{\prime \prime}$, the normalization condition becomes

$$
\begin{aligned}
N \mathcal{Z}= & \sum_{n_{1}^{\prime}} \cdots \sum_{n_{N}^{\prime}} e^{-\left(1 / k_{B} T\right)\left(\epsilon_{n_{1}^{\prime}}+\cdots+\epsilon_{n_{N}^{\prime}}\right)} \\
& \times\left\langle 0\left|\hat{c}_{n_{1}^{\prime}} \cdots \hat{c}_{n_{N}^{\prime}} \hat{c}_{n_{N}^{\prime}}^{\dagger} \cdots \hat{c}_{n_{1}^{\prime}}^{\dagger}\right| 0\right\rangle .
\end{aligned}
$$

Recalling Eq. (A4), the following identity is achieved

$$
\begin{aligned}
\sum_{s} \sum_{R} n_{s} e^{-\left(1 / k_{B} T\right) \epsilon_{R}}= & \sum_{n_{1}^{\prime}} \ldots \sum_{n_{N}^{\prime}} e^{-\left(1 / k_{B} T\right)\left(\epsilon_{n_{1}^{\prime}}+\cdots+\epsilon_{n_{N}^{\prime}}\right)} \\
& \times\left\langle 0\left|\hat{c}_{n_{1}^{\prime}} \ldots \hat{c}_{n_{N}^{\prime}} \hat{c}_{n_{N}^{\dagger}}^{\dagger} \ldots \hat{c}_{n_{1}^{\prime}}^{\dagger}\right| 0\right\rangle .
\end{aligned}
$$

The summations $\Sigma_{n_{1}^{\prime}}$ and $\Sigma_{s}$ are both summations over all the states of the confining potential; thus it is possible to change the index $s$ to $n_{1}^{\prime}$ to obtain the relation

$$
\begin{aligned}
\sum_{n_{1}^{\prime}} \sum_{R} n_{n_{1}^{\prime}} e^{-\left(1 / k_{B} T\right) \epsilon_{R}}= & \sum_{n_{1}^{\prime}} \cdots \sum_{n_{N}^{\prime}} e^{-\left(1 / k_{B} T\right)\left(\epsilon_{n_{1}^{\prime}}+\cdots+\epsilon_{n_{N}^{\prime}}\right)} \\
& \times\left\langle 0\left|\hat{c}_{n_{1}^{\prime}} \ldots \hat{c}_{n_{N}^{\prime}} \hat{c}_{n_{N}^{\prime}}^{\dagger} \ldots \hat{c}_{n_{1}^{\prime}}^{\dagger}\right| 0\right\rangle
\end{aligned}
$$

and

$$
\begin{aligned}
& \bar{n}_{n_{1}^{\prime}} \mathcal{Z}=\sum_{R} n_{n_{1}^{\prime}} e^{-\left(1 / k_{B} T\right) \epsilon_{R}}=\sum_{n_{2}^{\prime}} \ldots \sum_{n_{N}^{\prime}} e^{-\left(1 / k_{B} T\right)\left(\epsilon_{n_{1}^{\prime}}+\ldots+\epsilon_{n_{N}^{\prime}}\right)} \\
& \times\left\langle 0\left|\hat{c}_{n_{1}^{\prime}} \ldots \hat{c}_{n_{N}^{\prime}} \hat{c}_{n_{N}^{\prime}}^{\dagger} \ldots \hat{c}_{n_{1}^{\prime}}^{\dagger}\right| 0\right\rangle .
\end{aligned}
$$

The above equation tells us that the distribution functions for bosons and for fermions can be obtained not only with Eq. (A1) but also as

$$
\begin{aligned}
\bar{n}_{n_{1}^{\prime}}= & \frac{1}{\mathcal{Z}} \sum_{n_{2}^{\prime}} \ldots \sum_{n_{N}^{\prime}} e^{-\left(1 / k_{B} T\right)\left(\epsilon_{n_{1}^{\prime}}+\cdots+\epsilon_{n_{N}^{\prime}}\right)} \\
& \times\left\langle 0\left|\hat{c}_{n_{1}^{\prime}} \ldots \hat{c}_{n_{N}^{\prime}} \hat{c}_{n_{N}^{\prime}}^{\dagger} \ldots \hat{c}_{n_{1}^{\prime}}^{\dagger}\right| 0\right\rangle,
\end{aligned}
$$

where $N-1$ summations appear and all the summations are extended over all the states of the confining potential. It is the matrix element $\langle 0|\cdots| 0\rangle$ that selects the allowed configurations and provides the right number of particles for a given configuration. The one-particle reduced WF is obtained by performing the integrals over the Wigner variables of $N-1$ particles. Therefore, using Eqs. (7) and (10) and $N-1$ times Eqs. (A6)-(A8), the one-particle reduced WF reads

$$
\begin{aligned}
f_{W}^{(N)}\left(x_{1}, p_{1}\right)= & \frac{1}{\mathcal{Z}} \sum_{n_{1}^{\prime}} \sum_{n_{1}^{\prime \prime}} \sum_{n_{2}^{\prime}} \cdots \sum_{n_{N}^{\prime}} \\
& \times\langle 0| \hat{c}_{n_{1}^{\prime}} \hat{c}_{n_{2}^{\prime}} \cdots \hat{c}_{n_{N}^{\prime} \hat{c}_{n_{N}^{\prime}}^{\dagger} \cdots \hat{c}_{n_{2}^{\prime}}^{\dagger} \hat{c}_{n_{1}^{\prime \prime}}^{\dagger}|0\rangle} \\
& \times e^{-\left(1 / k_{B} T\right)\left(\epsilon_{n_{1}^{\prime \prime}}+\cdots+\epsilon_{n_{N}^{\prime}}\right)} \int d s_{1} e^{-(i \hbar) p_{1} s_{1}} \\
& \times \psi_{n_{1}^{\prime}}\left(x_{1}+\frac{s_{1}}{2}\right) \psi_{n_{1}^{\prime \prime}}^{\dagger}\left(x_{1}-\frac{s_{1}}{2}\right) .
\end{aligned}
$$

In the above equation, the mean value $\langle 0|\cdots| 0\rangle$ is equal to zero if $n_{1}^{\prime \prime} \neq n_{1}^{\prime}$, and is equal to the RHS of Eq. (A14) otherwise. Substituting Eq. (A14) into (A15) the one-particle reduced WF reads

$$
f_{W}^{(N)}\left(x_{1}, p_{1}\right)=\sum_{n_{1}^{\prime}} \bar{n}_{n_{1}^{\prime}} f_{W_{n_{1}^{\prime}}}\left(x_{1}, p_{1}\right) .
$$


${ }^{1}$ E. Wigner, Phys. Rev. 40, 749 (1932).

${ }^{2}$ W. Frensley, Rev. Mod. Phys. 62, 745 (1990).

${ }^{3}$ M. Nedjalkov, H. Kosina, S. Selberherr, C. Ringhofer, and D. K. Ferry, Phys. Rev. B 70, 115319 (2004).

${ }^{4}$ C. Jacoboni and P. Bordone, Rep. Prog. Phys. 67, 1033 (2004).

${ }^{5}$ P. Carruters and F. Zachariasen, Rev. Mod. Phys. 55, 245 (1983).

${ }^{6}$ F. Rossi and T. Kuhn, Rev. Mod. Phys. 74, 895 (2002).

${ }^{7}$ L. V. Keldysh, Zh. Eksp. Teor. Fiz. 47, 1515 (1964) [Sov. Phys. JETP 20, 1018 (1965)].

${ }^{8}$ M. Pascoli, P. Bordone, R. Brunetti, and C. Jacoboni, Phys. Rev. B 58, 3503 (1998).

${ }^{9}$ J. G. Kirkwood, Phys. Rev. 44, 31 (1933).
${ }^{10}$ C. Harper, Am. J. Phys. 42, 396 (1974).

${ }^{11}$ G. E. Uhlenbeck and L. Gropper, Phys. Rev. 41, 79 (1932).

${ }^{12}$ M. Hillery, R. F. O'Connell, M. O. Scully, and E. P. Wigner, Phys. Rep. 106, 121 (1984).

${ }^{13}$ K. Imre, E. Ozizmir, M. Rosenbaum, and P. F. Zweifel, J. Math. Phys. 8, 1097 (1967).

${ }^{14}$ E. Cancellieri, P. Bordone, A. Bertoni, G. Ferrari, and C. Jacoboni, J. Comput. Electron. 3, 411 (2004).

${ }^{15}$ F. Reif, Statistical and Thermal Physics (McGraw-Hill, New York, 1965).

${ }^{16}$ R. G. Chambers, Proc. Phys. Soc., London, Sect. A 65, 458 (1952). 